\title{
BIODEGRADACION DE PAJA DE TRIGO POR CEPAS FUNGICAS SELECCIONADAS
}

\author{
(Biodegradation of wheat straw by selected fungal strains)
}

\author{
Ricardo Cabeza ${ }^{1}$, Dante Pinochet ${ }^{1}$ y Eduardo Valenzuela ${ }^{2}$ \\ ${ }^{1}$ Universidad Austral de Chile, Instituto de Ingeniería Agraria y Suelos, \\ Facultad de Ciencias Agrarias, Casilla 567. Valdivia Chile. \\ ${ }^{2}$ Universidad Austral de Chile, Instituto de Microbiología, \\ Facultad de Ciencias, Casilla 167. Valdivia Chile.
}

Palabras claves: Biodegradación, paja de trigo, lignina, celulosa, Trichoderma pseudokonigii, Westerdikella multispora Key words: Biodegradation, wheat straw, lignin, cellulose, Trichoderma pseudokoningii, Westerdikella multispora

\section{RESUMEN}

Desde suelos derivados de cenizas volcánicas se evaluó la capacidad degradativa de 2 cepas fúngicas seleccionadas:Trichoderma pseudokoningii $y$ Westerdikella multispora y una cepa control (Trametes versicolor ) sobre paja de trigo previamente sometida a pasteurización y con adición de $\mathrm{NH}_{4} \mathrm{NO}_{3}$. Como controles fueron incluidos paja de trigo y paja de trigo más $\mathrm{NH}_{4} \mathrm{NO}_{3}$, ambas sin inóculo fúngico.

Todos los tratamientos fueron realizados en triplicado y mantenidos durante 60 días en un invernadero. Al inicio y al final de las incubaciones se determinó los contenidos de celulosa, lignina, extraíbles totales, $N, P, C$ totales, cenizas y $\mathrm{pH}$.

Los tratamientos controles no presentaron variación en los contenidos de celulosa. Las pajas tratadas con las cepas fúngicas presentaron una disminución no significativa del contenido de celulosa pero un aumento significativo del contenido de lignina. El contenido de extraíbles totales sólo en la paja tratada con T. pseudokoningii aumentó significativamente, la cual además presentó una disminución significativa del contenido de C. Por su parte, solo en las pajas tratadas con W. multispora se determinó un aumento significativo del N. Para el contenido de P total no se determinaron aumentos significativos.

En todos los tratamientos el pH disminuyó y las cenizas presentaron un aumento significativo en aquellos que incluyeron solo las cepas seleccionadas.

\footnotetext{
ABSTRACT

The decay ability of two selected fungal strains Trichoderma pseudokoningii and Westerdikella
}

multispora, and a control strains (Trametes versicolor) collected from volcanic ash soils wose evaluated on wheat straw which had been previously pasteurized and added with $\mathrm{NH}_{4} \mathrm{NO}_{3}$. As controls wheat straw and wheat straw plus $\mathrm{NH}_{4} \mathrm{NO}_{3}$ were included, both without fungi inoculation. All treatments were in three replicates and kept for 60 days in a greenhouse. At the beginning and at the end of the incubation period, the cellulose contents, lignin, extractable totals, $N, P, C$ totals, ashes and $\mathrm{pH}$ were determined. The control treatments did not alters cellulose content. Straws treated with fungal strains presented a non significant decrease on the cellulose content but a significant increase on the lignin content. The content of total extractable increased significantly only in the straw treated with T. pseudokoningii which also revealed a marked decrease in $C$ contents. In turn, only in straw treated with W.multispora there was a significant increase in $N$ content. The total $P$ content did not reveal any major increase. In all treatments with fungal strains $\mathrm{pH}$ decreased while ashes increased significantly in treatments involving only selected strains.

\section{INTRODUCCION}

Con el objetivo de buscar posibles alternativas a la quema de rastrojos dejados después de las cosechas de cereales, se estudió la capacidad de dos cepas fúngicas aisladas de un suelo trumao para biodegradar paja de trigo. El presente trabajo es la tercera parte de una serie de investigaciones llevadas a cabo en la Universidad Austral de Chile. La primera etapa consistió en aislar cepas fúngicas desde un suelo trumao y determinar su capacidad degradativa en condiciones de laboratorio (Olavarría, 2000). En la segunda etapa se determinó la capacidad 
degradativa de cepas seleccionadas en paja de trigo adicionada de Glucosa y $\mathrm{NH}_{4} \mathrm{NO}_{3}$ (Lindh, 2002, no publicado). En el presente ensayo se analizó la capacidad para biodegradar paja de trigo en forma natural de dos cepas fúngicas Trichoderma pseudokoningii y Westerdikella multispora, previamente aisladas desde un suelo trumao serie Pelchuquín comparándose con la cepa control Trametes versicolor CBS.

\section{MATERIALES Y METODOS}

Incubación de la paja de trigo con las cepas. Se usaron 2 cepas fúngicas, Trichoderma pseudokoningii (Rifai) y Westerdykella multispora (Saito \& Minoura) Cejp \& Milco, que fueron aisladas por Olavarria (2000), a partir de un suelo trumao serie Pelchuquín, y una cepa control, Trametes versicolor (L. Ex Fr). Cada una de las cepas se cultivo en forma independiente en agar extracto malta $2 \%$ (AEM 2\%) a $23^{\circ} \mathrm{C}$ durante 7 a 14 días. La paja de trigo fue cortada a un largo de $10 \mathrm{~cm}$, se pesó $1 \mathrm{~kg}$ de paja por tratamiento y repetición. Luego fue sometida a ebullición en 40 L de agua por 10 minutos, tras lo cual se dejó estilar hasta humedad de retención, a continuación fue homogeneizada y se mezcló con $\mathrm{NH}_{4} \mathrm{NO}_{3}(50,6 \mathrm{~g} / \mathrm{kg}$ de paja), luego se depositó en bolsas de nylon transparentes (40 x $54 \mathrm{~cm})$, y se inoculó con discos de agar con micelio de $1 \mathrm{~cm}$ de diámetro. Se depositaron 21 discos de agar con micelio por bolsa, más $116 \mathrm{~cm}^{3}$ de suspensión de micelios y esporas. Las bolsas sembradas se dejaron en un invernadero del centro experimental Santa Rosa de la UACH, por 60 días.

Determinación de la capacidad biodegradativa de las cepas. Al inicio y término de la incubación, como parámetros cuantitativos de la biodegradación de la paja de trigo, se procedió a determinar celulosa, lignina y extraíbles totales según los métodos propuestos por la norma TAPPI (1988), y N P C totales según Saavedra (1975), además cenizas y pH.

Diseño experimental. El diseño experimental consideró un período de 0-60 días con 12 muestras para cada tratamiento.

Como grupo control se utilizó:

Paja de trigo sin inóculo

Paja de trigo sin inóculo $+\mathrm{NH}_{4} \mathrm{NO}_{3}$

Paja de trigo $+\mathrm{NH}_{4} \mathrm{NO}_{3}+$ T.versicolor

Como tratamientos se utilizó:

Paja de trigo $+\mathrm{NH}_{4} \mathrm{NO}_{3}+\boldsymbol{T}$. pseudokoningii

Paja de trigo $+\mathrm{NH}_{4} \mathrm{NO}_{3}+\boldsymbol{W}$. multispora

Las fuentes de variación fueron: entre tratamientos al mismo tiempo y entre tiempo para igual tratamiento. Las comparaciones de promedio se realizaron me- diante la prueba de Tukey al 5\% de significancia.

\section{RESULTADOS Y DISCUSIÓN}

El mayor constituyente que conforma la paja de trigo es la celulosa con valores que oscilan entre los 48,90 a 51,02\%, para los tratamientos en el tiempo cero (Tabla 1). Los resultados coinciden con los obtenidos por otros autores, los cuales indican que la celulosa representa entre un 40-50\% del peso seco de la paja de trigo (Hoseney, 1986; Sylvia et al., 1999). Como segundo constituyente estructural de la paja se encuentra la lignina con valores que van desde un 18,50 a 21,06\% para el tiempo cero (Tabla 1), lo cual es semejante a los resultados obtenidos por Olavarria (2000) y Paul \& Clark (1996), que indican valores promedio de $21,48 \%$ y de 18 a $21 \%$ respectivamente para paja de trigo.

En los tratamientos en los cuales la paja de trigo fue inoculada con cepa control T.versicolor, y cepas seleccionadas T.pseudokoningii y W.multispora, la celulosa tiende a disminuir, aunque sin diferencias estadísticas (Tabla 1). En el ensayo, si bien existe una degradación de la celulosa esta no es significativa, lo que reafirma lo señalado por diversos autores que indican que un alto contenido de lignina impediría alcanzar una rápida degradación (Wild, 1992; Sylvia et al., 1999; Kumar \& Goh, 2000). Deacon (1988), señala que incluso en condiciones de laboratorio la degradación del complejo lignocelulósico es lento.

Analizando el constituyente lignina, se observó un aumento significativo de su contenido relativo para el tratamiento con la cepa seleccionada T. pseudokoningii y W. multispora (Tabla 1), lo cual indicaría una degradación de componentes carbonados y un aumento en los compuestos más resistentes en forma relativa, debido a la acumulación de polifenóles condensados que poseen tasas muy lentas de descomposición (Paul \& Clark, 1996).

Lo observado en el ensayo, indicó que la degradación de la paja de trigo corresponde a una disminución en la cantidad absoluta de celulosa para todos los tratamientos que incluyen alguna cepa fúngica, y a un aumento relativo en los mismos tratamientos para el constituyente lignina, en donde sólo los tratamientos con T. pseudokoningii y $\mathbf{W}$. multispora presentan un aumento significativo (Tabla 1). Esto indicaría el consumo de carbono en base a celulosa y una acumulación de lignina, con resultados similares a los obtenidos por Fengel \& Wegener (1983). Este consumo sostendría el crecimiento de los microorganismos, proporcionando energía y carbono para formar los nuevos constituyentes

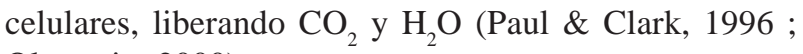
Olavarria, 2000). 
Tabla 1. Variación de constituyentes químicos-orgánicos en paja de trigo sometida a biodegradación con cepas fúngicas durante 60 días.

\begin{tabular}{|c|c|c|c|c|c|}
\hline $\begin{array}{l}\text { Días desde la } \\
\text { inaubación }\end{array}$ & Paja control sin inóculo & $\begin{array}{c}\text { Paja control sin inóculo + } \\
\mathrm{NH}_{4} \mathrm{NO}_{3}\end{array}$ & $\begin{array}{l}\text { Paja }+\mathrm{NH}_{4} \mathrm{NO}_{3}+ \\
\text { T. versicolor }\end{array}$ & $\begin{array}{l}\text { Paja }+\mathrm{NH}_{4} \mathrm{NO}_{3}+ \\
\text { T. pseudokoningii }\end{array}$ & $\begin{array}{c}\text { Paja }+\mathrm{NH}_{4} \mathrm{NO}_{3}+ \\
\text { W. multispora }\end{array}$ \\
\hline & \multicolumn{5}{|c|}{ Contenido de celulosa (g/100g) } \\
\hline 0 & $49,01 \pm 0,92 \quad$ (a) a & $48,90 \pm 6,60 \quad$ (a) a & $50,70 \pm 5,46 \quad$ (a) a & $51,02 \pm 0,82$ (a) a & $49,65 \pm 0,76 \quad$ (a) a \\
\hline \multirow[t]{2}{*}{60} & $51,03 \pm 0,57$ (b) a & $50,34 \pm 1,05$ (a) a & $48,32 \pm 1,32$ (a) a & $49,50 \pm 2,26$ (a) a & $48,79 \pm 1,02$ (a) a \\
\hline & \multicolumn{5}{|c|}{ Contenido de lignina (g/100g) } \\
\hline 0 & $19,94 \pm 0,90 \quad$ (a) a & $18,50 \pm 0,13 \quad$ (a) a & $21,06 \pm 1,74$ (a) a & $20,43 \pm 1,03$ (a) a & $21,00 \pm 0,11 \quad$ (a) a \\
\hline \multirow[t]{2}{*}{60} & $21,28 \pm 1,05 \quad$ (a) a & $23,44 \pm 0,85$ (b) bc & $21,97 \pm 0,40 \quad(a) a b$ & $24,30 \pm 0,64$ (b)c & $23,47 \pm 0,31 \quad$ (b) bc \\
\hline & \multicolumn{5}{|c|}{ Contenido de extraíbles totales (g/100g) } \\
\hline 0 & $10,62 \pm 1,09$ (a) a & $13,65 \pm 0,57 \quad$ (a) bc & $13,06 \pm 0,51 \quad$ (a)b & $14,37 \pm 0,47 \quad$ (a) bc & $15,25 \pm 0,76 \quad$ (a)c \\
\hline 60 & $10,48 \pm 2,48$ (a) $a$ & $14,68 \pm 0,89$ (a) $b$ & $14,02 \pm 1,21$ (a)b & $18,28 \pm 0,37$ (b) $c$ & $14,93 \pm 0,23$ (a) bc \\
\hline
\end{tabular}

Letras distintas entre paréntesis difieren significativamente a través de la columna para el parámetro medido y letras distintas sin paréntesis difieren significativamente en la fila para el parámetro medido $(\mathrm{P}<0.05)$.

*Cepa control y **Cepas seleccionadas.

Tabla 2. Variación de constituyentes químicos inorgánicos en paja de trigo sometida a biodegradación con cepas fúngicas durante 60 días.

\begin{tabular}{|c|c|c|c|c|c|}
\hline $\begin{array}{c}\text { Días descle la } \\
\text { inabación }\end{array}$ & Paja control sin inóculo & $\begin{array}{c}\text { Paja control sin inóculo + } \\
\mathrm{NH}_{4} \mathrm{NO}_{3}\end{array}$ & $\begin{array}{c}\text { Paja }+\mathrm{NH}_{4} \mathrm{NO}_{3}+ \\
\text { T. versicolor } \\
\end{array}$ & $\begin{array}{l}\text { Paja }+\mathrm{NH}_{4} \mathrm{NO}_{3}+ \\
\text { T. pseudokoningii* }\end{array}$ & $\begin{array}{c}\text { Paja }+\mathrm{NH}_{4} \mathrm{NO}_{3}+ \\
\text { W. multispora }^{* *}\end{array}$ \\
\hline & \multicolumn{5}{|c|}{ Contenido de nitrógeno (g/100g) } \\
\hline 0 & $0,27 \pm 0,02 \quad$ (a) a & $1,40 \pm 0,04 \quad(a) b$ & $1,53 \pm 0,20 \quad$ (a)b & $1,47 \pm 0,03 \quad$ (a)b & $1,45 \pm 0,05 \quad(a) b$ \\
\hline \multirow[t]{2}{*}{60} & $0,30 \pm 0,02 \quad$ (a) a & $1,72 \pm 0,07 \quad$ (b) $b$ & $1,68 \pm 0,12$ (a) b & $1,71 \pm 0,15$ (a)b & $1,61 \pm 0,08$ (b) $b$ \\
\hline & \multicolumn{5}{|c|}{ Contenido de fósforo (g/100g) } \\
\hline 0 & $0,0085 \pm 0,0011$ (a) ab & $0,0078 \pm 0,0001$ (a) ab & $0,0064 \pm 0,0014$ (a) a & $0,0093 \pm 0,0014$ (a)b & $0,0097 \pm 0,0005$ (a)b \\
\hline \multirow[t]{2}{*}{60} & $0,0085 \pm 0,0005$ (a) a & $0,0088 \pm 0,0014$ (a) ab & $0,0089 \pm 0,0013(a) a b$ & $0,0113 \pm 0,0003(a) b$ & $0,0106 \pm 0,0011(a) a b$ \\
\hline & \multicolumn{5}{|c|}{ Contenido de carbono (g/100g) } \\
\hline 0 & $41,44 \pm 0,60$ (a)b & $40,24 \pm 0,50$ (a) a & $40,00 \pm 0,28 \quad$ (a) a & $40,87 \pm 0,40 \quad$ (a) ab & $39,77 \pm 0,25$ (a) a \\
\hline 60 & $41,89 \pm 0,14$ (a) a & $40,44 \pm 0,33$ (a)b & $39,77 \pm 0,27 \quad$ (a)bc & $38,85 \pm 0,44$ (b) $c$ & $39,00 \pm 0,62$ (a)c \\
\hline
\end{tabular}

Letras distintas entre paréntesis difieren significativamente a través de la columna para el parámetro medido y letras distintas sin paréntesis difieren significativamente en la fila para el parámetro medido $(\mathbf{P}<0.05)$.

*Cepa control y **Cepas seleccionadas.

Los extraíbles totales, son el resultado de la suma de los extraíbles en etanol, etanol-tolueno e hidrosolubles. Se debe hacer mención que los extraíbles totales en el tiempo cero del ensayo son dispares, producto de lo heterogéneo de los extraíbles del material y de la aplicación de $\mathrm{NH}_{4} \mathrm{NO}_{3}$, mostrando sólo un aumento significativo la paja de trigo tratada con T. pseudokoningii, respecto de todos los demás tratamientos (Tabla 1). El aumento de los extraíbles totales para el tratamiento con la paja de trigo más T. pseudokoningii, coincide con el mayor aumento del componente lignina, lo que ratificaría lo expuesto por Hoseney (1986) y Flaig et al. (1977), quienes indican que los compuestos que se acumulan en la degradación no son ocupados como fuente de carbono debido a su constitución, la cual podría actuar como biocida y material inhibidor.

Para el parámetro nitrógeno total , la paja control sin inóculo al tiempo cero, presenta un promedio de 
Tabla 3. Variación de cenizas y del pH en paja de trigo sometida a biodegradación con cepas fúngicas durante 60 días.

\begin{tabular}{|c|c|c|c|c|c|}
\hline $\begin{array}{c}\text { Dias descle la } \\
\text { inabación }\end{array}$ & Pajacontrol sininóalo & $\begin{array}{c}\text { Paja control sininóalo + } \\
\mathrm{NH}_{4} \mathrm{NO}_{3}\end{array}$ & $\begin{array}{c}\text { Paja }+\mathrm{NH}_{4} \mathrm{NO}_{3}+ \\
\text { T. versicolor }\end{array}$ & $\begin{array}{l}\text { Paja+ }+\mathrm{NH}_{4} \mathrm{NO}_{3}+ \\
\text { T. pseuddkoningii }\end{array}$ & $\begin{array}{c}\text { Paja }+\mathrm{NH}_{4} \mathrm{NO}_{3}+ \\
\text { W.multispora }\end{array}$ \\
\hline & \multicolumn{5}{|c|}{ Conteridodecenizas (g/100g) } \\
\hline 0 & $2,90 \pm 0,12 \quad$ (a)c & $2,02 \pm 0,18 \quad(a) \mathrm{a}$ & $2,25 \pm 0,27$ (a)a & $2,38 \pm 0,12(\mathrm{a}) \mathrm{ab}$ & $2,85 \pm 0,15$ (a)bc \\
\hline \multirow[t]{2}{*}{60} & $2,81 \pm 0,34$ (a)ab & $2,39 \pm 0,19$ (a) a & $2,43 \pm 0,30$ (a)a & $2,66 \pm 0,11$ (b) ab & $3,15 \pm 0,11$ (b)b \\
\hline & \multicolumn{5}{|c|}{ pH } \\
\hline 0 & $6,16 \pm 0,06$ (a)b & $4,22 \pm 0,18 \quad$ (a)a & $4,33 \pm 0,27$ (b) a & $4,32 \pm 0,12$ (b)a & $4,22 \pm 0,15$ (b)a \\
\hline 60 & $6,45 \pm 0,29$ (a)b & $4,00 \pm 0,19$ (a) a & $3,92 \pm 0,30$ (a) a & $3,92 \pm 0,11$ (a)a & $3,89 \pm 0,11$ (a)a \\
\hline
\end{tabular}

Letras distintas entre paréntesis difieren significativamente a través de la columna para el parámetro medido y letras distintas sin paréntesis difieren significativamente en la fila para el parámetro medido $(\mathbf{P}<0.05)$. *Cepa control $\mathbf{y} * *$ Cepas seleccionadas.

0,27\% (Tabla 2), lo que coincide con los porcentajes cercanos al 0,3\% obtenidos por Olavarria (2000). Estos resultados concuerdan con los expresados por Szegi (1988), quien determinó un 0,24\% para paja de centeno, y Beare et al. (2002) con un 0,4\% para paja de cebada, las variaciones se deben principalmente a los estados nutricionales de las plantas y las condiciones de desarrollo del cultivo. Los resultados para los tratamientos que incluyeron cepas fúngicas y el tratamiento control sin inóculo adicionado de $\mathrm{NH}_{4} \mathrm{NO}_{3}$ presentan una cantidad relativa mayor para nitrógeno, debido a la adición de $\mathrm{NH}_{4} \mathrm{NO}_{3}$, variando entre 1,40 y $1,53 \%$ para los distintos tratamientos en el tiempo cero (Tabla 2). Sólo el tratamiento paja control más $\mathrm{NH}_{4} \mathrm{NO}_{3}$ y el tratamiento con la cepa $\boldsymbol{W}$. multispora presentaron una variación significativa de $\mathrm{N}$ total entre el inicio y final de la incubación. El aumento de nitrógeno indicaría que podría existir degradación, según lo observado por Olavarria (2000),en tanto Verano \& Burges (1989), indican que si existe pérdida de celulosa existiría un aumento de nitrógeno. La paja de trigo tratada con W. multispora, fue el único tratamiento que incorporó alguna cepa en donde existió aumento de nitrógeno con significancia estadística, coincidiendo en un aumento significativo de lignina.

Se observó la variación del fósforo total para los tratamientos sometidos a degradación, los valores obtenidos van desde 0,0064 a $0,0097 \%$ para el tiempo cero (Tabla 2), estos valores relativamente bajos se explican porque el material utilizado era esencialmente tallos, los cuales poseen menor concentración de fósforo que las hojas. Se observó una tendencia general para el fósforo a aumentar, sin embargo, en ninguno de los tratamientos se presentó diferencias estadísticas signifi- cativas.

Los valores de las concentraciones relativas de carbono para paja de trigo sometida a biodegradación durante 60 días oscilan entre un 41,44\% para el tratamiento paja control sin inóculo y un 39,77\% para el tratamiento que incluye la cepa $\boldsymbol{W}$. multispora (Tabla 2), estos coinciden con Collins et al. (1990a y 1990b) con un 41,4 y $42,7 \%$ para paja de trigo. De acuerdo a los valores de la Tabla 2, sólo el tratamiento que incluye la cepa T. pseudokoningii exhibe una disminución significativa de carbono respecto del tiempo cero, indicando degradación de la paja para este tratamiento. Collins et al. (1990a y 1990b), Madhulika et al., (1996) y Olavarria (2000), indican que una disminución del contenido total de carbono implicaría degradación, debido a que los microorganismos utilizan el carbono como fuente de energía, liberando $\mathrm{CO}_{2}$ en la respiración. También estos resultados coinciden con Fogarty \& Kelly (1990), quienes postulan que fracciones de celulosa son utilizados como fuente de energía y transformados a $\mathrm{CO}_{2}$ por microorganismos aerobios como bacterias y hongos.

Además de los parámetros químicos inorgánicos, se midieron la variación del $\mathrm{pH}$ de la paja de trigo y la cantidad de cenizas. Los contenidos relativos de cenizas para paja de trigo antes y después de ser sometidas a descomposición por microorganismos, son cercanos a los registrados por Olavarria (2000), alrededor de 3,9\% y son casi la mitad del contenido de cenizas obtenidos por Collins et al. (1990a y 1990b), para tallos de trigo con un 6,3\%. Estas diferencias se pueden deber a condiciones de suelo y estado nutricional del cultivo, lo que incidiría en el contenido de minerales absorbidos.

Para el tiempo cero del ensayo se observa una disminución significativa del $\mathrm{pH}$ en los tratamientos de 
partidas de paja adicionada de $\mathrm{NH}_{4} \mathrm{NO}_{3}$ (control sin inóculo adicionado de $\mathrm{NH}_{4} \mathrm{NO}_{3}$, control T. versicolor, $\boldsymbol{T}$. pseudokoningii y $W$. multispora), respecto del control sin inóculo, esta disminución se debe a la adición de $\mathrm{NH}_{4} \mathrm{NO}_{3}$, el cual posee una reacción ácida (Tabla 3). El $\mathrm{pH}$ de la paja (control sin inóculo) es cercano al pH neutro (6.16), el cual coincide con los resultados obtenidos por Olavaria (2000). Además se observa que todos los tratamientos que incluye alguna cepa fúngica presentan una disminución del pH entre el tiempo cero y 60 días de incubación en forma significativa, según Olavarria (2000), los hongos de importancia lignocelulósica prefieren ambientes ácidos, por lo tanto el $\mathrm{pH}$ que presenta el sustrato adicionado de nitrato de amonio sería óptimo, además el mismo autor señala que los hongos producto de su propio metabolismo acidificarían el medio, por lo tanto lo observado en el presente estudio para el parámetro $\mathrm{pH}$, coincide con antecedentes previos, además Kishan et al. (1993), indican que de un pH inicial de 9.82 disminuyó a 9.62-9.7 en paja de trigo, debido presumiblemente por la descomposición de productos lignocelulósicos.

\section{CONCLUSIONES}

Después de 60 días de incubación la paja de trigo tratada con nitrógeno fue parcialmente degradada por las cepas T. pseudokoningii y $\mathbf{W}$. multispora lo que se reflejó en un aumento en el contenido relativo de lignina y en un aumento relativo de los extraíbles totales sólo para la cepa T. pseudokoningii. No se observó un aumento en la concentración de celulosa, lo que indicaría una degradación de ella en forma proporcional a su contenido.

La cepa T. pseudokoningii fue superior a la cepa control en la degradación de la paja de trigo ya que presentó una disminución en el contenido relativo de carbono después de la incubación, en conjunto a un aumento en los contenidos de lignina y extraíbles totales.

Las cepas seleccionadas, $T$. pseudokoningii y $W$. multispora tuvieron una mayor actividad biológica sobre la paja de trigo en comparación a la cepa control $\boldsymbol{T}$. versicolor lo que se determinó por una disminución en el pH del sustrato y un aumento del contenido de cenizas

\section{AGRADECIMIENTOS}

Los autores agradecen el financiamiento de este trabajo a la Universidad Austral de Chile (proyecto DIDUACH 200210).

\section{REFERENCIAS}

Beare, M.; Wilson, P.; Fraser, P. \& Butler, R. (2002). Management effects on barley straw decomposition, nitrogen release, and crop production. Soil Science Society of American Journal. 66: 848-856

Collins, H. ; Elliot, L.; Rickman, R.; Bezdicek, D. \& Papendick, R. (1990a). Decomposition and interactions among wheat residue components. Soil Science Society of American Journal. 54: 780-785

Collins, H.; Elliot, L. \& Papendick, R. (1990b). Wheat straw decomposition and changes in decomposability during field exposure. Soil Science Society of American Journal. 54: 1013-1016

Deacon, J. (1988). Introducción a la micología moderna. Editorial Limusa S.Ade C.V. México.

Fengel, D. \& Wegener, G. (1983). Wood chemistry ultrastructure reactions. Walter Gruyter \& Co. Berlín, Alemania.

Flaig, W. Nagar, B.; Söchting, H. \& Tieitjen, C. (1977). Organic materials and soil productivity. Food and Agriculture Organization of the United States. Roma, Italia. Bulletin 35

Fogarty, W. \& Kelly, C. (1990). Microbial enzymes and biotechnology. Ed. Departament of Industrial Microbiology. London, England.

Hoseney, R. (1986). Principles of cereal. Science and technology. Ed.
American association of Cereal Chemists. Minnesota, USA.

Kishan, S.; Singh, G. \& Gupta, B. (1993). Effect of Phanerochaete crysosporium inoculation on in vitro digestibility and chemical composition of wheat straw. Indian Journal of Dairy Science. 46(4): 177 179

Kumar, K. \& Goh, M. (2000). Crop residues and management practices: Effects on soil quality, soil nitrogen dynamics, crop yield, and nitrogen recovery. Advances in Agronomy. 68: 197-319

Madhulika. A.; Singh, D. \& Malik, R. (1996). Effect of carbon sources on degradation of wheat straw by lignocellulolytic fungi. Environment and Ecology. 14 (3): 612-614

Olavarria, G. (2000). Caracterización enzimática cualitativa de cepas fúngicas de un suelo trumao y determinación mediante parámetros químicos de su capacidad para biodegradar paja de trigo. Tesis de Licenciado en Ciencias Agrarias. Valdivia. Universidad Austral de Chile. Facultad de Ciencias Agrarias.

Paul, E. \& Clark, F. (1996). Soil microbiology and bichemistry. $2^{\circ}$ Ed. Academic Press. San Diego, CA., USA.

Saavedra, N. (1975). Manual de análisis de plantas. Pontificia Univ. Católica de Chile. Facultad de Agronomía. Publicación número 17. pp 17-36 
Sylvia, D.; Fuhrmann, J.; Hartzel, P. \& Zuberer, D. (1999). Principles and applications of soil microbiology. Prentice Hall. Uppe Saddle River, New Jersey, U.S.A.

Szeigi, J. (1988). Celulose decomposition and soil fertility. Ed. Akadémai Kaidó. Budapest, Hungary.

Tappi,S. (1988). Test methods tecnology. Park, Atlanta, USA. Vol. I.
Verano, A. \& Burgess, L. (1989). Decomposition and composition chemistry of cereal straw. Applied Microbiology and Biotechnology. 21 (4): 551-559

Wild, A. (1992). Condiciones del suelo y desarrollo de las plantas según Russell. Ediciones Mundi Prensa. Madrid, España. 